\title{
Assessing the transferability of a hybrid Taguchi-objective function method to optimize image segmentation for detecting and counting cave roosting birds using terrestrial laser scanning data
}

\begin{abstract}
As far back as early 15th century during the reign of the Ming Dynasty (1368 to 1634 AD), Gomantong cave in Sabah (Malaysia) has been known as one of the largest roosting sites for wrinkle-lipped bats (Chaerephon plicata) and swiftlet birds (Aerodramus maximus and Aerodramus fuciphagus) in very large colonies. Until recently, no study has been done to quantify or estimate the colony sizes of these inhabitants in spite of the grave danger posed to this avifauna by human activities and potential habitat loss to postspeleogenetic processes. This paper evaluates the transferability of a hybrid optimization image analysis-based method developed to detect and count cave roosting birds. The method utilizes high-resolution terrestrial laser scanning intensity image. First, segmentation parameters were optimized by integrating objective function and the statistical Taguchi methods. Thereafter, the optimized parameters were used as input into the segmentation and classification processes using two images selected from Simud Hitam (lower cave) and Simud Putih (upper cave) of the Gomantong cave. The result shows that the method is capable of detecting birds (and bats) from the image for accurate population censusing. A total number of 9998 swiftlet birds were counted from the first image while 1132 comprising of both bats and birds were obtained from the second image. Furthermore, the transferability evaluation yielded overall accuracies of 0.93 and 0.94 (area under receiver operating characteristic curve) for the first and second image, respectively, with $\boldsymbol{p}$ value of $<\mathbf{0 . 0 0 0 1}$ at $\mathbf{9 5 \%}$ confidence level. The findings indicate that the method is not only efficient for the detection and counting cave birds for which it was developed for but also useful for counting bats; thus, it can be adopted in any cave.
\end{abstract}

Keyword: Taguchi-objective; Wrinkle-lipped bats; Swiftlet birds 
